\title{
Advertising's Misinformation Effect
}

\author{
Kathryn A. Braun, University of lowa \\ Elizabeth F. Loftus, University of Washington
}

This research explores whether post-experience advertising alters information learned in a consumer's direct experience. An advertising misinformation effect was obtained for color memory of a previously seen candy bar wrapper upon both visual and verbal misinformation. However, the misleading visual information produced more 'remember' judgments than misleading verbal information. This advertising misinformation effect did not dissipate when the source was discredited. We found that such memory changes can be directly linked to consumer subjective judgments and choices when the misleading information is particularly salient. Not only do these findings constitute a novel generalizability of the misinformation effect, they also have implications for social policy research on deceptive advertising. (c) 1998 John Wiley \& Sons, Ltd.

Advertising is far from impotent or harmless; it is not a mere mirror image. Its power is real, and on the brink of a great increase. Not the power to brainwash overnight, but the power to create subtle and real change. The power to prevail. (Clark, 1985)

Advertising is part of creating product illusion, and sometimes that illusion process can go beyond its intended boundary. A case in point is Listerine ${ }^{\mathrm{TM}}$, whose parent company Warner-Lambert was sanctioned by the Federal Trade Commission (FTC) for producing misleading advertising that led some consumers to believe their product was an effective preventative for colds (Armstrong, Gurol and Russ, 1979; Harris and Monaco, 1978). Since that time, social policy researchers have been studying how advertising can be miscomprehended by consumers to lead to the creation of false product beliefs (Burke et al., 1988; Jacoby and Hoyer, 1982). While the creation of false beliefs may be important, they might not be the only illusion manufactured by advertising.

What if the Listerine ${ }^{\mathrm{TM}}$ commercial caused consumers to falsely remember something they had learned during their own past experience with the product? From reconstructive memory research we know that false memories can seem real, even if they differ significantly from the experience being 
remembered. The rich perceptual detail that characterizes remembered past personal experiences is very compelling and can produce an intuitive sense that memories of this experiential quality must be veridical (Schacter, 1995). Because consumers prefer to believe they are relying on own experiences when making decisions (Deighton, 1984), advertising's influence on consumer product memories may go unnoticed and thus have an insidious effect on behavior. This can be seen as an example of a sourcemonitoring error, in which the information is remembered but the source of the memory (internal versus external) is confused (Johnson, Hashtroudi and Lindsay, 1993).

Although the FTC sanctioned Listerine ${ }^{\mathrm{TM}}$ for leading some consumers to believe that their product could prevent colds, it never assessed whether the advertising led consumers to believe that they themselves had in the past been cured of a cold by the mouthwash. In other words, the FTC found that the advertisement changed some individuals' beliefs about the product, but did not explore whether it also changed consumers' memory for their experiences with that specific product. In this research we examine the malleability of memory for product information by looking at whether misinformation in an advertisement received after a direct experience with a product can become part of consumers' memory.

It is well known that most advertising is for 'mature' products, that is, products that consumers have already experienced. We also know that advertising for products that consumers have already purchased is especially likely to be noticed (Lodish et al., 1995), and much theorizing exists to explain why this might be so (e.g. Cohen and Goldberg, 1970 offer a dissonance reduction explanation). However, none of this discussion concerns the possibility that advertising might have a retroactive effect and change the way a past experience with the product is remembered. We refer to this phenomenon as the 'advertising misinformation effect'. The term comes, of course, from an adaptation of the more general 'misinformation effect' in cognitive psych- ology, where it has been repeatedly shown that misleading information presented after a previously experienced event can change the way people remember the past event (e.g. Loftus, 1977; Belli et al., 1994).

This research explores whether misleading advertising about a previously experienced product can change people's memory for the color of the product's wrapping. Although the color of the wrapping might seem to be a rather trivial detail, the color of food packaging is considered to be extremely important in the marketing world (Sloan, 1996). Colors not only enrich sensory product experiences, they also convey information to consumers about a product's image. Searching for package color is a common heuristic used by consumers, and any distortion of color information could lead consumers to buy the wrong product. Moreover, color information is sometimes deceptively 
manipulated by product competitors, who have been known to create 'copy cat' wrapping to mimic the color scheme of a category leader. This latter color deception has already caught the attention of the Supreme Court (Barrett, 1995).

The suggestibility of color memory can be tested using a color wheel. This measure is sensitive to memory blending because it contains responses that include: (1) the original color viewed, (2) the suggested color, and (3) colors that are intermediate between the two. Thus, one can examine the extent to which people will come to misremember a response that is a blend of two colors, and the extent to which they will come to misremember a response that is simply the more extreme suggested color. Some past studies (e.g. Belli, 1988) have reported relatively few blended memories, while other have reported more (e.g. Loftus, 1977). Perhaps this has something to do with procedural differences between these studies.

In this research we adapt the traditional color shift paradigm that has been used before to distort memory for the colors of objects (Loftus, 1977; Belli, 1988). In one of the older color memory studies, respondents were shown a series of slides depicting an auto-pedestrian accident in which a green car drives by the scene (Loftus, 1977). Immediately following the slideshow respondents answered a series of questions. A 'misled' group received a question suggesting that the passing car was blue, while the control group did not receive color misinformation. When tested later, many of the respondents who had received the misleading color information misremembered the color of the passing car in a way that was aligned with the misinformation. That is, many remembered a 'blue' or 'bluish-green' car.

In the first study we present here, respondents viewed a green wrapper around a chocolate bar during what they thought was a product taste test. Later, they saw an advertisement suggesting that the wrapper was blue. Finally, subjects were tested on their memories for the color of the product that they had previously experienced.

In experiment 1, we manipulated not only whether respondents received misleading blue color information but also whether the misinformation was presented within the advertisement visually or verbally. A few studies have manipulated the type of misinformation (visual versus verbal) when examining the more traditional misinformation effect. In one study (Pezdek, 1977) subjects were presented with a sequence of pictures and sentences, followed by intervening pictures and sentences, and finally a recognition test. When misleading intervening items were presented, recognition accuracy for the original items was suppressed. This effect occurred despite the fact that the intervening items were presented in a different modality from the earlier to-be-recognized items. 
Our manipulation of the modality of the misinformation in Experiment 1 was straightforward. In the visual case, the misinformation was presented by showing an advertisement depicting the candy in a blue wrapper. In the verbal case the misinformation was presented by describing the candy as appearing with a 'blue' wrapper. In both cases we hypothesized that the misinformation may become semantically integrated in memory, although subsequent memory for color might be different in the two cases. In the visual case respondents might ultimately remember a color that was a close match to the color seen in the advertisement, where the misinformation overrides the original information due to its perceptual similarity. In the verbal case respondents might commonly misremember the color as one that was a blend between the color they had experienced (green), and the color described ('blue'), and consequently remember a bluish-green (Loftus, 1977). To assess whether these two types of misinformation memories were phenomenologically different, we asked whether respondents actually 'remembered' seeing the reported color, or whether they simply 'knew' it to be the color, following suggestions of Tulving (1985). This distinction between remembering and knowing has been found to arise from a post- recollective process where respondents attend to the product of their retrieval and has been adapted by other researchers in recognition tests for studying false memories (Roediger and McDermott, 1995).

Experiment 2 explores whether warning respondents that they may have been exposed to misinformation lessens the advertising misinformation effect. Traditional misinformation studies have examined the impact of warnings, and most tend to find that the placement of the warning makes a difference (Greene, Flynn and Loftus, 1982; Wright, 1993). Warnings that come too late, after the misinformation has had ample time to contaminate, typically have little influence. In Experiment 3, we conducted a survey to assess the effect of color on consumer decision making. In Experiment 4 we looked at whether our color change would be associated with meaningful subjective evaluations of the product. We explored this issue by falsely suggesting to respondents, in a fairly subtle fashion, that green wrappers contain a dye that is potentially harmful, while blue wrappers have been found to be safe, and then let them rate the relative safety of the new product that they had previously experienced.

\section{Experiment 1}

The major goal of Experiment 1 was to explore misinformation effects in an advertising context. Some respondents received misinformation about the color of a critical product, a chocolate bar that actually had a green wrapper. Some respondents received misinformation that the color was blue, but they received the misinformation visually as a picture of the bar in the print advertisement; others 
received the misinformation verbally in the advertisement copy. All respondents were sub- sequently tested on their memory for the color of the product wrapper. We hypothesize that misinformation in the form of advertising would lead respondents to misremember their experiences with the chocolate bar-coming to recall a blue or bluish-green color. In addition, this experiment assessed whether the modality of the misleading information affects how the target information is recollected.

\section{Method}

\section{Participants}

The participants were 96 undergraduates ( 32 female, 64 male) who received course credit for their involvement in this study. Ten male participants who reported that they were color-blind were excluded from the analysis.

\section{Design and Procedure}

A single-factor design was used where the type of post-experience information was varied across three levels (control, no misinformation; visual; misinformation as a picture; and verbal, misinformation as verbal suggestion). A timeline illustrating the critical time points for the various experimental events appears in Figure 1.

Respondents came to the experimental setting expecting to participate in a chocolate taste test. They were run in groups ranging from 15 to 25 . They were told that Suisse (the target bar, identified by a fictitious brand) would be challenging Godiva for the upscale chocolate buyers market. The experimenter passed around samples of the Suisse product to each respondent on a large platter which contained a large $8 \times 4$-inch Suisse bar with a green wrapper. All respondents had to see the bar to get their chocolate sample. Respondents rated the Suisse chocolate for taste. The same procedure was followed for the Godiva chocolate (which had a gold box). The experimenter thanked respondents for their input and another experimenter came to deliver a 15-minute filler task.

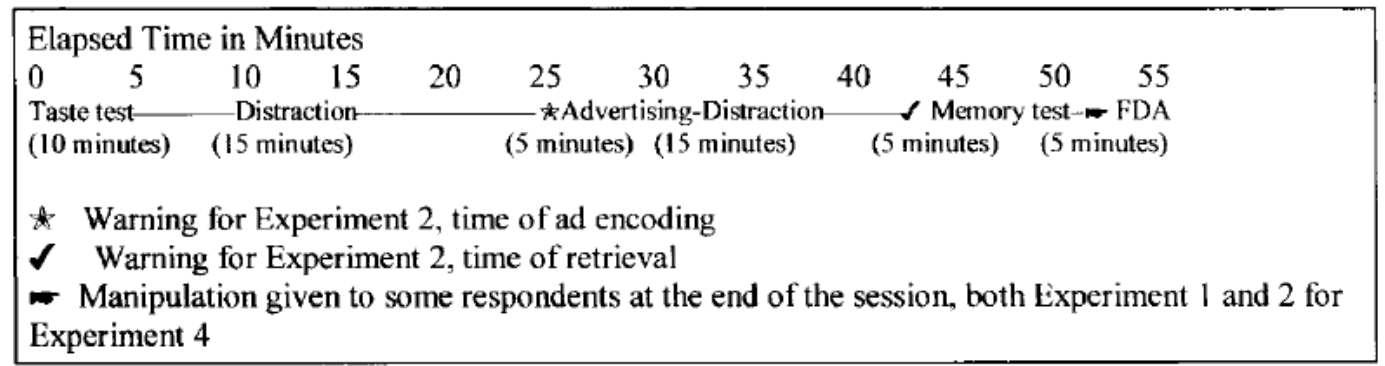

Figure 1. Timeline of the experiments 
The main experimenter returned and stated Suisse was planning to launch an advertising campaign against Godiva. Respondents were asked to evaluate Suisse and Godiva print advertisements, and were instructed to think about whether Suisse was properly positioning themselves against Godiva. The Godiva advertisement was seen first. They were then given one of three Suisse advertisements depending on experimental condition. The advertisement copy read: 'when you crave a chocolate candy bar, look for: Suisse (control); this wrapper (and a picture of a blue Suisse bar)'; or 'the blue wrapper that says Suisse'.

After another 15-minute distracter task respondents were tested on their memory for the taste test. The critical part was a color recognition test, where they were asked to match what they remembered from objects in the taste test against a color wheel. This wheel had 30 discrete colors representing additive primary, secondary and their blended colors. Each color had an associated numeric value. Ten objects were included; the Suisse wrapper was the target item. ${ }^{1}$

After they identified the color of each object, we applied the procedure developed by Tulving (1985) in which respondents distinguish between states of awareness about the past: remembering and knowing. Further, a 'guess' option was offered in case respondents had no memory for the object. They were told to indicate a judgment as 'remember' if their memory for the object involved a visual reexperiencing of it, that they could picture the object (and its color) in their mind's eye. They were told to indicate a memory judgment as a 'know' judgment if it was based more upon recognition or familiarity (that somehow one of the colors seemed appropriate but that they could not picture the object itself). And they were told to indicate a memory judgment as a 'guess' if they randomly selected one of the 30 colors as the object color, and that they had no recollection of the objection nor its color from the taste test. They made these judgments for all ten objects used as memory items on the test.

\section{$\underline{\text { Results }}$}

\section{Color memory}

The first analysis explored whether misinformation influenced respondents' color memory. To reiterate, the final color memory test required respondents to select the color of the chocolate bar wrapper by choosing a number corresponding to a color on the color wheel. Their responses ranged

\footnotetext{
${ }^{1}$ The color memory test consisted of the following ten items which respondents had seen during the taste test (the true colors appear in parentheses): dixie cups (peach); napkins (pink); felt on tray (purple); Godiva box (gold); Suisse wrapper (green); water bottles (blue); cracker box (red); doily cups (yellow); mint stripe (green); flag on wrapper (red).
} 
from 1 to 30, the numbers associated with the individual color strips on the wheel. These responses appear in Figure 2.

Of the 26 control respondents who did not receive any misinformation about the green wrapper, 19 (73\%) selected a green, represented by either color strip 14 or 15, both medium greens closest to the original color wrapper. Other reports from this control group included 7 (27\%) selecting an unrelated color, e.g. red or yellow. No one in this group selected a shade of blue, represented by color strips $5-8$, or a bluish-green color, represented by color strips $9-12$. Of the 31 respondents in the verbally misled group, 10 (32\%) selected a green color. The other responses given by the verbal misled respondents were 11 blue (35\%), 4 (13\%) bluish-green, and 16 (19\%) random. Of the 29 respondents in the visual misled group (represented by the thick solid line), 10 (34\%) selected a green. The other responses given by the visual misled group were $13(45 \%)$ blue, 2 (7\%) bluish-green, and 4 (13\%) random. Both misled conditions were compared to the control group using the Mann-Whitney procedure for ordinal data. Both misled groups were significantly closer to the misleading blue than the control group, $z=3.37, p<0.001$ for the verbal misled versus control; $z=3.73, p<0.0001$ for the visual versus the control. There was no difference in rank shifts between the visual and verbal groups, $z<1 .^{2}$ Thus, as the memory-impairment hypothesis would predict, the control respondents were more likely than both misled groups to accurately select the correct green color. The misled groups were more likely to choose an incorrect color, and especially to choose a blue or bluish-green hue, indicating an influence of the misinformation.

\footnotetext{
${ }^{2}$ An alternative way to analyze the data would be to subtract the accurate color (14) from the reported color to produce a composite shift measure. A shift near ' 0 ' would indicate no effect of misinformation, a negative shift would indicate a misinformation effect. Using ANOVA with experimental condition as the independent measure and the shift as the dependent measure, the overall model was significant: $F(2,83)=6.88, p<0.001$. Using the Tukey post-hoc comparisons with $p=0.05$, both the visual $(M=-2.0)$ and verbal $(M=-1.87)$ were significantly different from the control ( $M=3.5)$ but not significantly different from one another. The critical difference was 3.375, MSE=38.94. This was also done without the random responses (e.g. red) to reduce the MSE. Again, the overall model was significant, $F(2,69)=10.96, p<0.001$. The same results were obtained with Tukey post-hoc tests, where both visual $(M=-4.04)$ and verbal $(M=-3.43)$ were significantly different from the control $(M=0.368)$ but not one another. The critical difference was $3.38, \mathrm{MSE}=10.87$.
} 


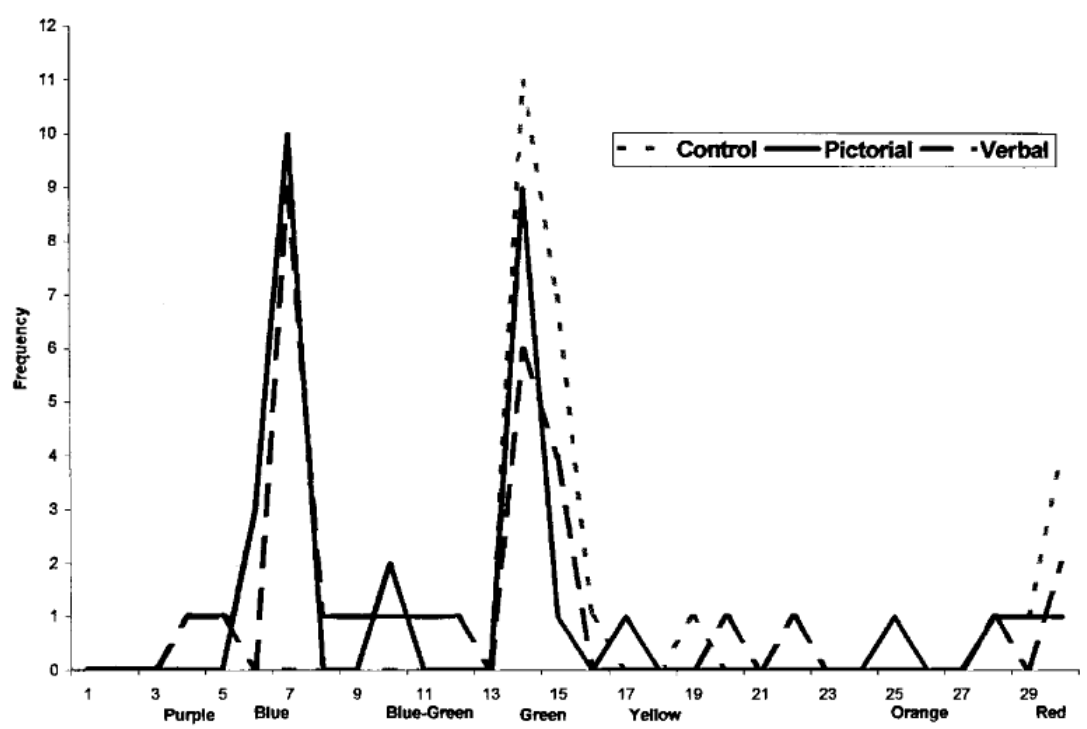

Figure 2. Experiment 1: frequency distribution of colour memory by condition

A more detailed analysis comparing the verbal and visual misinformation was conducted. For this analysis we classified responses into four categories: correct green, misinformation color blue replacement; blend color; bluish-green; and random other. We found more memory blending reported in the verbal condition (13\%) than the visual condition (7\%), but this did not reach statistical significance, $\chi^{2}<1$; and more replacement in the visual condition (45\%) than the verbal condition (35\%), but again, not statistically different, $\chi^{2}<1$. There was also no significant difference in the proportion of misled versus accurate responses in the visual versus verbal conditions. In fact, there was more blending/distortion, in the direction of the verbal condition-61\% misled in verbal, $50 \%$ misled in visual, but not significantly so, $\chi^{2}<1$.

\section{Type of Memory Judgments}

We looked at the phenomenological qualities of both the misled and accurate memories using Tulving's remember/know distinction, where 'remember' refers to a visual re-experiencing of the chocolate bar, e.g. seeing a 'blue' color in their mind's eye; 'know' is generally recognizing the blue color; and we added a 'guess' response to indicate if they had just randomly guessed and had no feeling of knowing the wrapper's color. A distribution of these judgments by experimental condition appears in Figure 3. To examine the misled memories, we looked at 30 respondents who reported blue or bluishgreen responses for the Suisse bar. Overall, we found the majority of misled memories to be either 'remember' (33\%) or 'know' (55\%), with only $12 \%$ indicating they were guessing. There were differences in the phenomenological qualities of the misled judgments between the two misled conditions, suggesting that the modality of the misinformation had an influence on the ecphory of the memory- 
retrieval experience. There are significantly more 'remember' judgments in the visual condition (47\%) than in the verbal condition $(19 \%), \chi^{2}(1, n=30)=2.76, p<0.05$. This large number of 'remember' responses in the visual condition indicates that while semantic meaning of visual and verbal misinformation may be the same, there is an episodic component of recall for which visual misinformation seems to have more influence.

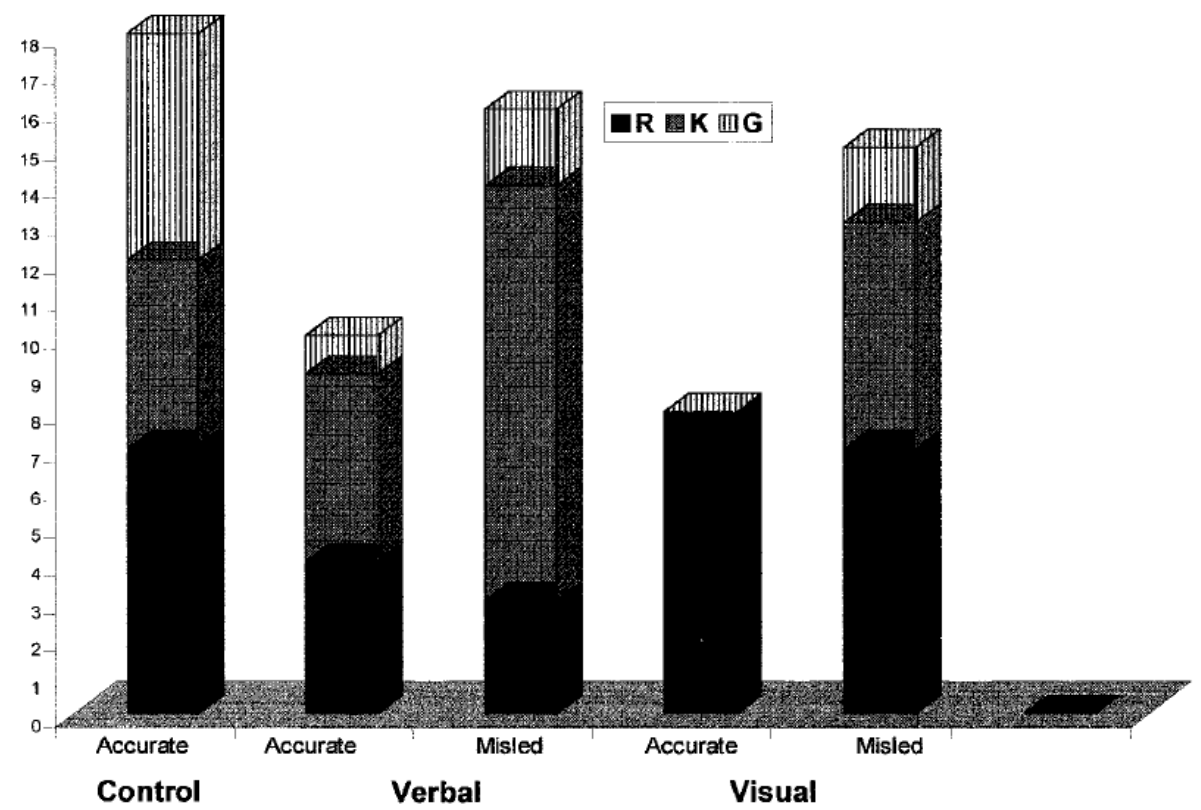

Figure 3. Experiment 1: phenomenological characteristics of memory judgements by condition

Further evidence of this visual misinformation's effect on the recall experience was determined by looking at the accurate memories. As with the misled respondents, the visual condition had significantly more 'remember' judgments than the verbal condition, $100 \%$ visual versus $40 \%$ in the verbal condition, $\chi^{2}(1, n=20)=7.2, p<0.007$. In addition, the accurate group that received the visual misinformation had significantly more 'remember' judgments than those accurate in the control condition, $100 \%$ visual versus $38 \%$ control, $\chi^{2}(n=26)=8.47, p<0.004$.

To test whether the misled results were driven by respondents with faulty memories, we looked at accuracy rates for the other nine memory test items. These accuracy rates did not differ significantly between groups, $82 \%$ for the control group, $78 \%$ for the visual misled group, and $79 \%$ for the verbal misled group. ${ }^{3}$

\footnotetext{
${ }^{3}$ Specifically, memory for Godiva, the competitor whose advertisement was seen by all respondents (and did not mention the color of the box), averaged $75 \%$ accuracy. There were no significant differences across experimental groups. The phenomenological qualities of the Godiva box were about the same breakdown as Suisse had been in
} 


\section{$\underline{\text { Discussion }}$}

The major finding from this study was that post-experience misinformation in the form of advertising can alter consumer memory for an event experienced. Specifically, recollection of the color of the wrapping on a previously tasted chocolate was influenced by misleading information contained in the subsequent advertisement about the chocolate. Both visual and verbal misinformation affected this recognition memory; however, the 'remember' responses to rate to the erroneous color was greater when the misinformation was presented visually than verbally.

This experiment replicates previous finding that the modality of the intervening information is not as critical as the semantic meaning contained (e.g. Pezdek, 1977). Lockhart (1979, p. 82): 'The distinction between sensory and semantic codes has in an important sense been drawn too sharply. There is a tendency to regard the sensory component of a word or pictures as a kind of detachable skin within which is a kernel of meaning but that is itself meaningless. Sensory and semantic features do not possess this simple additive relationship. The sensory codes are aspects of analysis of meaning.' At some level, the meaning of both the visual and verbal misinformation is infiltrating memory, we see high misinformation effects for both conditions. At another level, though, the modality of the misinformation matters, affecting the phenomenological aspect of retrieval. Tulving (1985) says the phenomenon or remembering is determined jointly by semantic and episodic information.

This distinction is important for two reasons. First, the majority of research in the misinformation effect has not distinguished between these two states of knowing (Roediger and McDermott, 1995). Second, the remember/know distinction provides greater insight on how different modalities might affect the reconstruction process. Those who received the visual misinformation really believed in the veracity and strength of the newly created memories \pm they reported visually reexperiencing the information. 'Remember' responses are supposed to be given if respondents could 'picture' the object (and its color) in their mind. Perhaps the visual misinformation enabled respondents to experience just that kind of 'picture' as they were viewing the advertisement. In doing so, some respondents may have realized that the actual experience with the wrapper had been different from what the advertisement suggested. In this respect the visual misinformation may have been more conspicuous, serving as a retrieval guide both to displace and strengthen the original color memory. As evidence, the bimodal distribution for this group consists of about half- and-half accurate-green and misled-blue responses and within both are high reported 'remember' memories. Since the memory test 
was in the visual modality this may have heightened the quality of recollective experience by serving as a more substantial retrieval cue for both the accurate-green and the previously seen misled-blue (Pezdek and Greene, 1993; Yamashita, 1996).

The purpose of this study was to test the generalizability of past misinformation findings to an advertising context. Both the Loftus (1977) eyewitness study and this advertising experiment showed high misinformation effects (defined as blending or replacement) $-48 \%$ for Loftus's original study, $50 \%$ for our visual group, $61 \%$ for our verbal group. But the advertising and the eyewitness testimony environment differ in several ways. There seemed to be more replacement from the advertisement information, defined as reporting a 'blue' memory. In the eyewitness case, Loftus (1977) found 30\% of the respondents reported a blue color (defined as 6-8 on her wheel), while in our advertising study we found $42 \%$ replacement (also defined as 6-8 on the wheel). Loftus (1977) found primarily blending (62\%) of the two colors (9-12 on her wheel), while we found relatively little blending, $10 \%$ (defined as $9-13$ on our wheel).We also found more random memories of the target information (18\%) than in the Loftus (1977) experiment (10\%).

Our findings resemble Belli's results in showing low rates of blending and high rates of replacement. In addition, Belli (1988) found that a 'typicality' effect, where colors thought to be more prototypical also made intrusions into the recollected memory. The random responses in this study were not so random, with $77 \%$ of these random reports indicating 'red' to be the color of the Suisse wrapper. These random 'red' responses appeared mostly to be less strongly held than the misled or accurate memories: 8 (62\%) 'guesses'; 3 (23\%) 'know'; and 2 (15\%) 'remember'. The majority of these intrusions (54\%) occurred within the control group. This may be due to the fact that this color appears to be most 'typical' of a chocolate bar available on the color wheel. We investigate this typicality explanation in Experiment 3.

\section{Experiment 2}

Following the demonstration of an advertising misinformation effect in Experiment 1, it was natural to ask whether respondents could resist this memory contamination if they were warned that there might be problems with the color as reproduced, or warned that they might be getting misinformation in the advertising. This experiment has important social policy consequences: the usual corrective action adjudicated against deceptive advertisers is to require that they inform consumers that previously given information was incorrect. Do such warnings work? 
Warnings have been studied in the eyewitness domain (e.g. Greene, Flynn and Loftus, 1982), with reports that warnings have no effect on the misled judgments if given at the time of the memory test, but do reduce the misinformation effect if given at the time of presenting the misleading information. It has not yet been investigated how these warnings effect the ecphory of the retrieval process. Some researchers believe that the remember/know distinction may only be a proxy confidence measure (Donaldson, 1996). According to this view we might expect overall more know and guess responses. Others have found that the remember/know distinction represents two distinct routes to knowing and are differentially affected by manipulations (Rajaram, 1993). In the present case, a warning may be discounted if one really could 'remember' seeing the color of the wrapper in their mind's eye and not reduce or change reportings of this type. On the other hand, for those who report just knowing responses, such a warning may have a greater impact.

In this experiment we warned respondents by telling them that the color printer had malfunctioned so that some colors in the advertisement were not representative of the true colors, and that they should read the advertisement for its overall content, and not for aesthetic qualities.

Respondents were given this warning either at the time of encoding (the advertisement viewing), or just before the time of retrieval (the memory test). Based on previous research we might expect the timing of the warning to matter.

\section{Method}

Participants

Forty-two undergraduates (11 female, 31 male) received course credit for participating in this experiment. Five male color-blind respondents were omitted from the analysis.

Design

A single-factor between-subject design was used with two levels of the variable timing of the warning (at advertisement encoding, at time of retrieval). Respondents were randomly assigned to one of these two warning conditions. Only the visual misinformation condition was used in this study.

\section{Procedure}

The same procedure of Experiment 1 was followed except for warning of the color problem, which occurred either at the time of advertisement encoding or immediately before the memory test. 


\section{$\underline{\text { Results }}$}

\section{Color Memory}

We first examined whether the warning had an impact on respondents' reported color memories for the Suisse wrapper. The distributions of these responses appear in Figure 4. There are two distributions. Consider first, respondents given the warning at the time of advertisement encoding. Of these 18 respondents, 4 (22\%) reported an accurate green. The other responses were $6(33 \%)$ blue and 8 (44\%) bluish green. We compared these results to the visual misinformation condition of Experiment 1 where no warning was given. There were significantly more 'misled' responses, $78 \%$ misled at advertisement encoding versus $50 \%$ visual with no instructions, $\chi^{2}(1, n=47)=3.19, p=0.07$. This warning appeared to lead to a blending pattern similar to the eyewitness results.

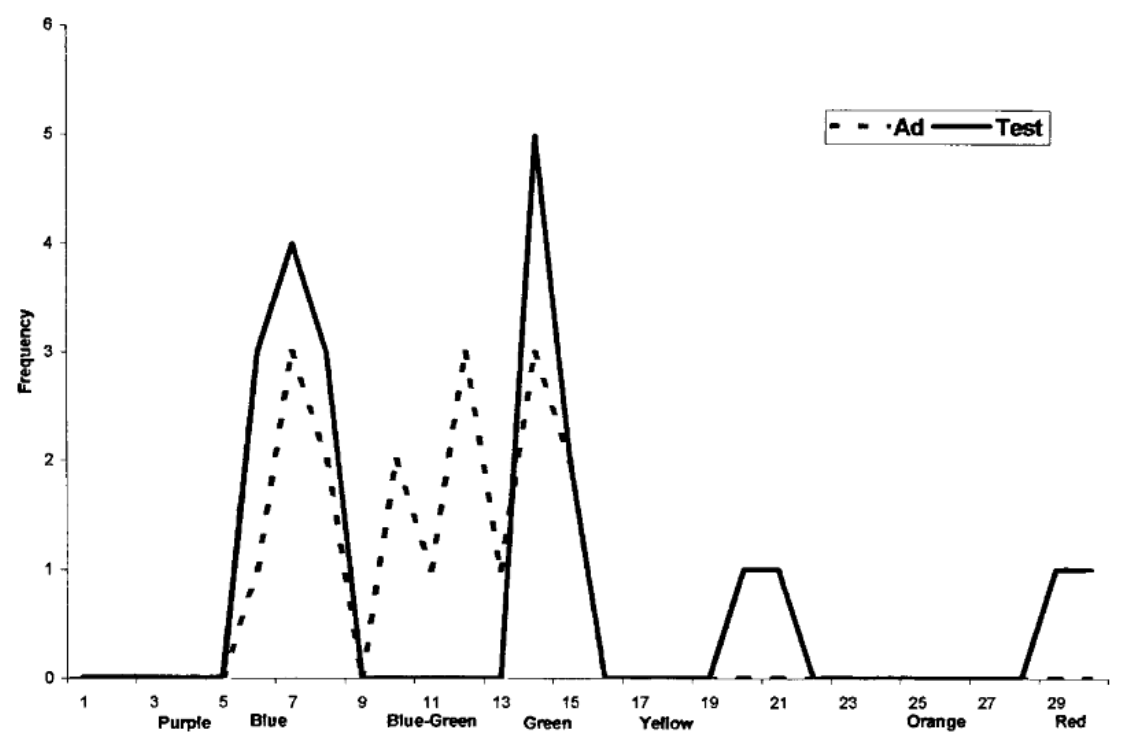

Figure 4. Experiment 2: frequency distribution of colour memory by warning condition

However, of the 19 respondents given the warning at the time of test, 6 (32\%) reported an accurate green, 10 (53\%) reported blue, 0 reported bluish-green, and 3 (15\%) reported another color. These results are very similar to those found in the visual condition of Experiment 1, resulting again in more replacement. There was no significant difference between the number of misled responses between this condition and that of Experiment 1, 50\% visual, no warning, 52\% warning at time of memory test, $\chi^{2}<1$.

We compared the means from both warning conditions to the control group from Experiment 1 to see whether there was a significant movement towards the color presented in the misleading advertisement. As in Experiment 1, both misled conditions differed significantly from the control group, 
$z=4.7, p<0.0001$ for warning at advertisement encoding, $z=3.06, p<0.001$ for warning at memory test. These two warning groups did not significantly differ from one another nor did either significantly differ from the no-warning visual condition of Experiment 1, both $z<1$. Therefore, the misinformation affected color memory in spite of the warning. ${ }^{4}$

What appeared to be the most interesting result, however, was in the different shapes of the distributions. We compared the distributions using the empirical distribution function (EDF) analyses for non-parametric data. We found no differences overall between the warning at encoding, warning at test, or visual with no warning distributions. However, because the differences among these groups appeared to be coming from the misled section of the graphs (whether there was either more blending or replacement), we analyzed the distributions of only the misled respondents in these conditions. Within this analysis the distributions did differ. Using the Kolmogorov-Smirnov test the warning at advertisement encoding distribution (which appeared more blended) was significantly different from the visual with no warning condition, $\mathrm{KSa}=1.37, \mathrm{p}=0.04$; and significantly different than the warning at test condition, $\mathrm{KSa}=1.67, \mathrm{p}=0.1$. There were no statistically significant differences between the warning at test and the visual with no warning distributions, both appearing to have more replacement responses.

\section{Type of Memory Judgments}

We looked at whether the warning affected how the color information came to be recalled. The remember/know/guess breakdown of both accurate and misled memory judgments appears in Figure 5. Consider first the 'misled' responses. For both the warning at encoding and at test there were significantly more 'guess' judgments in the encoding warning condition (69\%) than the $13 \%$ 'guess' rate in the no warning visual group of Experiment $1, \chi^{2}(n=28)=9.12, p<0.003$. The no-warning condition also significantly differed from the $40 \%$ 'guess' rate in the retrieval warning condition, $\chi^{2}(n=25)=2.34$,

\footnotetext{
${ }^{4}$ We performed using ANOVA on the composite shift measure for Experiment 2. With condition (control, no misinformation; visual, warning at advertisement encoding; and visual, warning at retrieval) as the independent variables and the shift as the dependent variable, the overall model was significant, $F(2,61)=9.31, p<0.0003$. Using the Tukey procedure for post-hoc tests, both the warning at encoding $(\mathrm{M}=-3.33)$ and the warning at retrieval $(\mathrm{M}=-$ 1.57) were significantly different from the control $(M=3.96)$, but not from one another. The critical difference was 3.39 and the MSE 35.82. As earlier, this was also done removing the random responses but did not change anything (other than allowing the means to reflect differences due to the misinformation rather than random choices, $M=-3.33$ for warning at encoding, $M=-4.25$ for warning at test.) We also looked at whether the shift due to the warning differed from the no warning visual condition of Experiment 1. Using ANOVA, with condition (visual, no warning; visual, warning at encoding, and visual, warning at retrieval) as the independent variables and the shift as the dependent variable, the model was not significant $(F<1)$ and no planned comparisons reached significance either, 3.39 was the critical difference (MSE 36.16). We also did this analysis removing the random responses, but still failed to find any differences.
} 
$p<0.1$. However, the number of 'remember' responses did not differ significantly from the no-warning group in either case, 31\% 'remember' at advertisement encoding condition, 30\% 'remember' at memory test, and $46 \%$ 'remember' with no warning, all $\chi^{2} 1$. It appears that the warning affected the 'know' judgments, turning them into 'guesses' while leaving the 'remember' judgments intact. This supports the view that these judgments represent two routes to 'knowing' rather than mere confidence. We note that the warning did affect how the accurate memories came to be remembered, with more 'guesses' and 'knows' reported than in Experiment 1. However, the number of accurate responses was too small ( $n=4$ for warning at encoding, $n=6$ for warning at time of test) to assess any statistical differences in the phenomenological experience for those memories.

\section{Accuracy Rates}

As in Experiment 1, the accuracy rate on the nine non-target test items was high for both groups: $80 \%$ for warning at the time, and $74 \%$ for the time of advertisement group. ${ }^{5}$ As a strong test of memorial differences between those who were exposed to misleading information and those who were not, we looked at whether there might be a difference for overall accuracy by each experimental group (including the cells from Experiment 1). There was not $F(4,119)=1.17, p=0.32$.

We also looked at whether people who had reported accurate memories for the Suisse bar differed from those who reported misled responses or random responses. Again, we found no difference, $F(2,121)=1.46, p=0.24$. Accuracy for the 'correct' Suisse wrapper response group for the other nine test items $(n=52)$ was $80 \%$, for the blended/'misled' group ( $n=59)$ was $77 \%$, and for the random response group $(n=13)$ was $80 \%$.

\footnotetext{
${ }^{5}$ Godiva's accuracy rate was the same as in Experiment 1,75\% for both conditions, but with more reported 'remember' $70 \%, 20 \%$ 'know' and 10\% 'guess'. The warning thus increased accuracy for the competitor's product.
} 


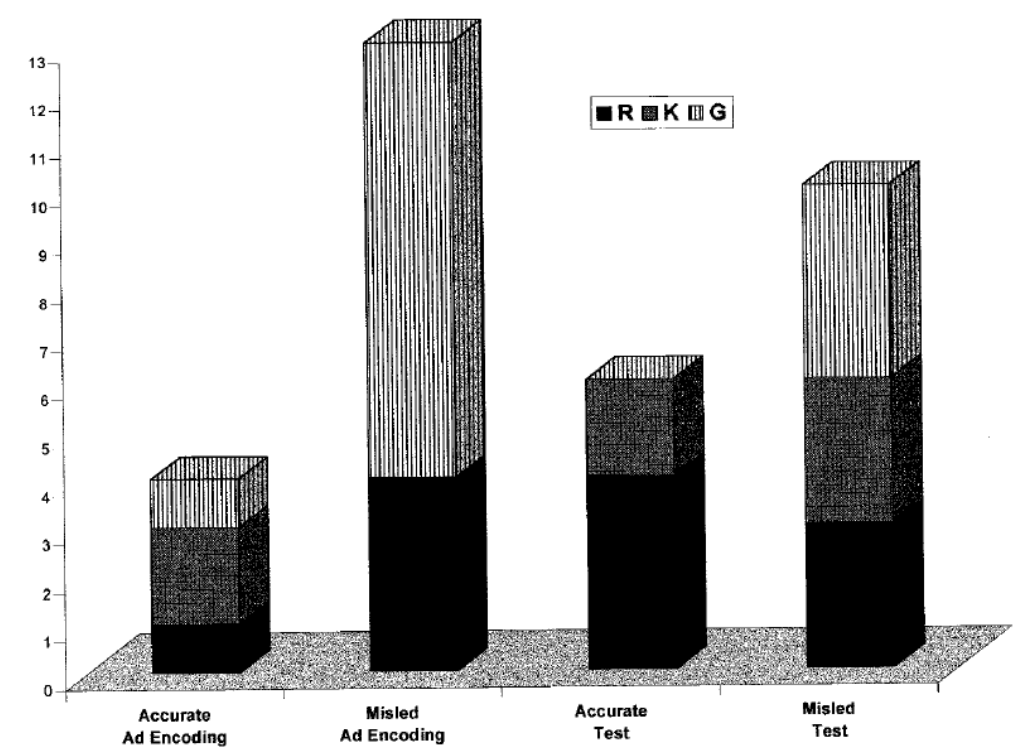

Figure 5. Experiment 2: phenomenological characteristics of memory judgements with warning

\section{$\underline{\text { Discussion }}$}

In this study we assessed whether warning respondents about the unreliability of the color information in the advertisements would reduce the misinformation effect found in Experiment 1 . We found it did not. However, the timing of the warning did matter. Respondents who were warned at time of advertisement encoding showed more misled responses in the form of blends. This warning altered how the respondents came to remember their experience, resulting in more 'guesses'. This is consistent with past research that has found reduced misinformation effects with warnings at the time of presentation because respondents spend longer scrutinizing the information (Tousignant, Hall and Loftus, 1986). In that study a 'blended' response was not available. Perhaps the blend (or compromise) responses in the present experiment came about because of indecision (or confusion), as evidenced by the large number of 'guess' judgments.

The finding of no effect of warnings at retrieval suggests that consumers are not knowingly relying on the advertisement to create memories of information gained in their direct experience or providing us with compromise judgments. It suggests that warning consumers about past fraudulent advertising messages might prove ineffective, especially if done after the information has already been incorporated into memory.

The phenomenological qualities of the misled memories confirmed previous research on the independence of the remember versus know distinction: only know responses seemed to be influenced by the warning. The number of remember responses was virtually the same in both Experiments 1 and 
2. And although the misled memory judgments in this study contained more reported guesses when given the warning, it is statistically improbable that this result was purely due to guessing. The misled area of the chart represented $(1 / 5)$ of the color wheel. It is probably more likely that the warning rendered the respondents who might have reported know responses for their metamemory judgments less certain, and this was expressed via the 'guess' option.

\section{Experiment 3}

The previous two experiments explored why consumers may not be aware of advertising's influence post-experience. The next part of the investigation identified the implications of the advertising misinformation effect with a survey to identify when color is important in consumer choice. Are some colors favored more by consumers than others? What colors are thought to be most 'typical' of candy bars?

\section{Method}

Participants

One hundred and seven undergraduates took the color survey. Seven color-blind males were omitted from the analysis.

\section{Design and Procedure}

Respondents were shown the color wheel used in the previous experiments and were asked to refer to it on their responses on the printed questionnaire. Embedded in a number of questions on color preference, the key measures of interest were:

\section{Typicality}

Which color do you think is most 'typical' of a candy bar wrapper? (number)

\section{Color Wrapper Preference}

How would you rate a candy bar with a color green (14) wrapper?

(anchored by 1 = poor, 5 = excellent)

How would you rate a candy bar with a color blue (6) wrapper?

(anchored by 1 = poor, 5 = excellent)

Would you prefer a candy bar with color '14' (green) or color '6' (blue) wrapper? 


\section{Color and Buying Behavior}

Would you ever buy a candy bar that had a wrapper color that was not appealing to you? $\mathrm{Y} \quad \mathrm{N}$ Imagine you are in the grocery store and searching for your favorite candy bar on the shelf. What is the first thing you look for in finding your bar? (open ended)

What first catches your attention when you see a new brand of candy bar on the shelf:

(Circle one)
a) its price
b) its color wrapper
c) its brand name
d) its size

other:

\section{$\underline{\text { Results }}$}

\section{Typicality}

We thought that the high intrusion of red in Experiment 1 might be due to a typicality effect, similar to what Belli (1988) found. In this survey, a reddish color was chosen as the most 'typical' of a candy bar the majority of the time-when asked about candy bar color typicality, $78 \%$ chose a 'red'; $10 \%$ an 'orange'; 6\% a 'yellow'; 3\% a 'blue' and 2\% a 'green'. Therefore, the blue/green intrusion reported earlier was most likely due to the misinformation rather than expectation and the 'random' red due to prior typicality expectations.

\section{Color Preference}

In our studies we changed respondents' memory from green to blue. Was this a change towards a positive? We found most people (74\%) would prefer a blue candy bar over a green one. And they rated a blue candy bar as significantly more favorable, $M=3.28$ versus $M=2.71, t(99)=3.5, p<0.0005$.

\section{Color and Buying Behavior}

Twenty per cent said they would never buy a candy bar that had a color wrapper they did not like, one said 'I'd be nervous eating something that looked strange on the outside'. Another said 'the outside of the wrapper makes me hungry and if I don't like the color, I'm not hungry anymore'. Most indicate, though, that there were more important determinants of their preference, such as taste. 
However, of those that said they would still buy an unattractive bar, $38 \%$ said they would do so only if they had heard good things, or tasted the bar themselves; that an unattractive color would deter them from otherwise buying.

In free recall, the most common search heuristic for finding their favorite bar in the store was searching by color, mentioned by $62 \%$ of respondents, second came brand name, mentioned by $26 \%$ of respondents. Color was also one of the first things respondents said they notice about a new candy bar, identified by $40 \%$ of them; brand name captured another $40 \%$; ingredients mentioned by $13 \%$ as a write- in in the 'other' slot; $4 \%$ identified price; and $4 \%$ identified size.

\section{$\underline{\text { Discussion }}$}

This survey found color to be an important heuristic consumers employ when searching store shelves. It is also one of the first things they notice about a new product. In addition, an unfavorable wrapper color may prevent initial product sampling. Color can also carry meaning about the product for consumers. We found that in our studies we moved respondents in the right direction as far as color wrappers, blue is much preferred over green. One respondent said: 'green reminds me of mould'. Another, 'green reminds me of being sick'. Others associated the color green with healthy products (perhaps due to the popularity of the Healthy Choice2 line or association with leafy vegetables), and not an appropriate color for a good-tasting candy bar wrapper. Last, and perhaps most important, we verified our previous assertion that the high incidence of reporting of red in Experiment 1 (and 2) was due to its typicality.

\section{Experiment 4}

While color may not be the most salient aspect in consumer choice, we believe that when such an objective trait carries semantic meaning for the consumers the memory change will be tied to judgment and behavior change. In this experiment we made the targeted color salient for the consumer by stating that the FDA had found that products with green labels were found to be contaminated and that blue or bluish-green were quite safe'. We expect that if consumers really changed their memory of the wrapper color then their safety judgments and choices between the two tasted candies (Godiva and Suisse) would also differ. We suspect consumers who are misled by the advertising information would report more favorable judgments about the Suisse bar and be more likely to choose it over Godiva when given a choice. 


\section{Method}

Participants

For this experiment we used 62 respondents ( 17 female, 35 male) who had participated in Experiments 1 and 2. These respondents were drawn from each of the five cells in Experiments 1 and 2 (9 from the control condition, 10 from visual, 12 verbal, 13 warning at advertisement, 18 warning at retrieval). This manipulation followed the measures reported in both earlier experiments.

Procedure

Following the memory test we presented respondents with information intended to manipulate the salience and the meaning they attached to the color of the wrapper. The purpose of this paragraph was to create an association between the color of the bar's wrapper with the product's putative safety. Recall that the actual color of the Suisse bar was green, which our FDA information said was unsafe, while the misleading advertising suggested the color blue, a safe color. Therefore, we expected respondents who had changed their memory to report that the bar was safer than Godiva. To avoid a ceiling effect where Godiva would always appear to be safer because of its genuine consumer reputation, we added some uncertainty to the choice by casting doubt on its safety, too. The following warning was read by respondents:

The FDA has noticed a high correlation between the color of food wrappers and the instance of food contamination. Wrappers which contain a yellow dye are particularly harmful. This dye is often used in foreign products. The FDA is particularly concerned about wrappers that are predominantly green, yellow, gold and orange. Meanwhile, laboratory tests have found that food wrappers which are predominantly bluish-green, blue and purple are quite safe. There has always been some health risk associated with products, like Godiva, which are perishable.

\section{Dependent Measures}

The respondents were then asked to rate the Suisse chocolate bar on the following three dimensions using continuous line mark scales (100 mm):

1. How safe do you think the Suisse product is compared to other chocolate products? (anchored by 'not very safe' and 'very safe')

2. What are your overall feelings toward the Suisse candy bar? (anchored by 'not very favorable' and 'very favorable') 
3. What is the likelihood you would buy this Suisse candy bar in the future?

(anchored by 'not very likely' and 'very likely')

4. If you had to buy a chocolate for a family member that was particularly susceptible to food contamination, which chocolate would you buy? (Circle one) Godiva Suisse

$\underline{\text { Results }}$

Respondents were divided into `accurate' memories and 'misled' groups. There were no significant differences between the experimental conditions so we collapsed across those groups. Seven respondents who reported random answers to the color test (e.g. red) were not used in this analysis. The accurate group consisted of 20 respondents who, in the color test, had correctly indicate the bar to be the true green color (either marking 14 or 15). The misled group consisted of 35 respondents who reported a bluish-green or blue color response in the memory test for the Suisse wrapper.

As expected, we found that the misled group perceived the Suisse bar to be significantly safer $(M=71$ for misled, $M=63$ for accurate), $F(1,53)=3.94, p<0.05)$; had significantly more favorable feelings ( $M=74$ for misled, $M=67$ for accurate), $F(1,53)=3.69, p<0.05)$; and were significantly more likely to state future buying intentions of the Suisse bar ( $M=62$ for misled, $M=51$ for accurate), $F(1,53)=2.64, p<0.05$ ); and, when given a choice between Suisse and Godiva, would be significantly more likely to choose Suisse (M. $74 \%$ for misled, M. $54 \%$ for accurate, $\left.\chi^{2}(1, n=55)=4.7, p<0.05\right)$.

\section{$\underline{\text { Discussion }}$}

The purpose of this experiment was to demonstrate that a change in color memory can directly affect consumers' subjective judgments and choice decisions. Because consumers may not be aware of the advertising's influence on their memory, one way post-experience advertising may work to provide information that may alter how a consumer's prior experience comes to be remembered. Since most advertising is intended to persuade and create positive attitudes, such misinformation is likely to lead to more favorable product memories. However, in some cases it can be deceptive as in the present case, causing consumers to have unwarranted feelings of safety toward the Suisse bar.

\section{General Discussion}

In two experiments we showed that misinformation received following a direct experience with a product altered the recollections respondents made about that product. In Experiment 1 we showed that respondents who saw a green wrapper on a chocolate bar and who later received misinformation 
that the wrapper on a chocolate bar was blue were more likely to remember a blue or bluish-green wrapper than respondents who did not receive misinformation. Misinformation influenced the recollections of the chocolate wrapper whether it was presented verbally or visually in the postexperience advertising. However, the visual information affected the phenomenological aspect of recall. Experiment 2 explored whether warning respondents that they might have received misinformation lessened the advertising misinformation effect. In general, a warning had minimal effects, rendering respondents less certain about their memory judgments if the warning was received at the time of encoding. Warnings given later when respondents were trying to retrieve information about the past had no effect.

In two later experiments we explored the effects of such advertising misinformation.

Experiment 3 found that while color may not be the most important attribute in chocolate bar selection, it does play an important role as a search heuristic within the grocery store. In Experiment 4 we investigated whether misinformation about the color of a chocolate wrapper could have consequences for a highly meaningful judgment about the chocolate. Respondents who received misinformation about the color of the wrapper, and who also were told that the misinformation color was associated with less contamination and greater safety, went on to make more favorable judgments about the critical chocolate bar and to indicate a great inclination to buy the chocolate in the future.

These findings extend and enhance our understanding of the misinformation effect more generally, and also extend the misinformation effect to a new context, namely the world of consumers and advertising.

\section{Implications for the Misinformation Effect}

Prior research produced evidence that misinformation can influence recollections of an event when it is in a dissimilar as well as similar modality from the event (e.g. Pezdek, 1977), but the stimuli used in that research were relatively pallid (sequences of pictures and sentences). Here, with a more realistic type of information processing setting and materials, we demonstrate the generalizability of Pezdek's (1977) findings; visual and verbal misinformation resulted in similarly high rates of memory distortion.

Prior work on the misinformation effect has not made a distinction between states of awareness about the past. False recognition due to misinformation has been attributed to familiarity, rather than an actual change in the memory (criticism of misinformation tests appear in McCloskey and Zaragoza, 1985). However, if this were the case, we would expect mostly know or guess responses than remember 
for the misled memories. The present experiments reported that those who had been misled by the advertising had memories that seemed real, with many reporting remember- type judgments. False memories can be remembered just as veridical ones can (Roediger and McDermott, 1995).

Others have looked at test modality as a means of correcting the misinformation effect, where when the modality of the misinformation is the same as the test there is greater misinformation effect and when the test is in the same modality as the original there is less (Pezdek and Greene, 1993; Yamashita, 1996). It is thought that modality serves as a retrieval cue either helping the recall of misinformation or the original to-be-remembered information. We advance this investigation by focusing on the type of intervening information and how that later comes to be recalled at the time of test. There appears to be more going on than the test serving as an activating cue for these memories. For instance, the verbal misinformation showed effects on the visual test to the same degree as the visual misinformation. The phenomenological aspects of recall also provide some insight into the process. Not only did the visual misinformation produce more remember judgments than the misled respondents of the verbal misled group, it also did so for the accurate respondents, producing more 'remember' than the verbal group. And, most interesting, more remember responses than were found in the control group. If the test instrument merely allowed greater access to the original color information we would expect that the control group would have as many remember responses as the visual misled group. Therefore, it seems that the visual misinformation had a role in creating these resultant judgments. Perhaps this vivid information 'pushed' respondents towards better remembering the accurate green color or replacing the color completely with the new misinformation. The bimodal distribution showing half-accurate and half- replaced color memories provides some indication this may be happening. In related work, Roediger and McDermott (1995) found that asking respondents to recall information shortly after exposure resulted in more subsequent remember judgments, both for accurate and inaccurate memories. Within our study the visual misinformation may have served as a rehearsal cue (for both the accurate-green and misled-blue wrapper) and become better accessed with the visual test instrument, a more applicable retrieval cue, and resulting in respondents reporting they had visually re-experienced the past (Pezdek and Greene, 1993).

Another critical issue in the misinformation field concerns the extent to which misled respondents simply adopt the misinformation as-is (replacing the original information with the misinformation), or whether they blend the misinformation with the original information to produce some 'intermediate'-type of response. Our findings also bear out this issue. We found more replacement responses than blend responses, a finding similar to Belli (1988). Most of our memory 
distortions were attributable to the misinformation itself (Loftus, 1977) but we also found some due to expectations of what color a typical chocolate wrapper should be (Belli, 1988). The latter intrusion resulted in more 'guesses' and less 'remembering' than did the misinformation. Both prior knowledge and induced misinformation have been shown to influence memory (Loftus, 1982). What is worth noting here is that the phenomenological aspects between these two intrusions differed, with the misinformation leading to more strongly-held memories.

The ability of respondents to benefit from warnings that they may be exposed to misinformation represents another critical issue in the misinformation literature. A typical finding is that warnings at the time of processing misinformation do allow respondents to benefit from the warnings, and to resist the misinformation better (e.g. Greene, Flynn and Loftus, 1982; Christiaansen and Ochalek, 1983). Warnings given very soon after exposure to misinformation also have been shown to have some positive effects (e.g. Wright, 1993; Wright, Varley and Belton, 1996). However, warnings given much later, sometimes have little or no effect (e.g. Greene, Flynn and Loftus, 1982; Lindsay, 1990).

We, too, found a differential effect of the warning dependent on when the respondents received it. If the respondent was warned at the time the advertisement was encoded there was some effect. It barely reduced the misinformation effect, but it did tend to make respondents less certain of their memory judgments. When the warning occurred very close to the advertisement misinformation it is possible that respondents have both accurate and misleading information available to them. In fact, this is the conclusion of Wright (1993, p. 153): 'multiple traces may exist for a limited period, but not indefinitely.' Perhaps this has something to do with why many respondents produce a blended response ('bluish-green'). They may be saying to themselves 'What color did I see if I thought it was green, but the advertisement calls it blue? A bluish-green?'

The delayed warning, on the other hand, had little effect. Moreover, most of the errors that respondents made were replacement errors - they chose a good solid blue rather than a bluish-green. Perhaps too much time had passed and the original color representation was no longer available to them, having been overwritten by the misinformation. Respondents in this group felt less sure about their judgments, but since they didn't have access to the original color, their responses were the same as if they had not been warned.

We found that warnings also influenced the distribution of remember/know judgments. The remember memory judgments were not impacted by our warning, only the know memory judgments seemed to be. These judgments were probably related to confidence, as Donaldson (1996) has found. This disassociation suggests that it may get at other aspects of remembering, as well (Rajaram, 1993). 


\section{Implications for Consumers and Advertising}

This paper began with an example of advertising as an illusion, and how the FTC punished a deceptive advertiser for pushing this illusion too far. Deception can occur when consumers are misled to believe that the advertised brand has a particular benefit. Or it can occur by convincing them that a competitor's product lacks something it might actually have had. In the past researchers have looked at deceptive advertising's effect on currently held beliefs. The present research demonstrates that naturally occurring misinformation may be planted to make consumers believe that past experiences contained information other than what was actually experienced.

Since the 1970s the FTC has required deceptive advertisers to 'correct' past mistakes through subsequent advertising messages, supposedly a benefit to consumers and competitors. Whether this correction can work retroactively is debatable: 'Corrective advertising has "worked". But not nearly well enough to even approach correcting the misimpression levels in the marketplace' (Wilkie, McNeill and Mazis, 1984, p. 26).

Perhaps the FTC needs to require corrections to be more explicit. For instance, in this study we were vague regarding the misinformation, maybe a more explicit warning such as 'this advertisement contains false information about the color of the Suisse bar. It is green. It is not the blue you saw in the advertisement' may help eliminate the effects. Christiaansen and Ochalek (1983) found that only explicit warnings were effective in reducing misinformation effects.

From the present research, we suggest one reason for the ineffectiveness of corrective advertising is that it is coming too late. Consumers may have already incorporated the information into their own belief system or be misattributing the deceptive message to another source, such as their own experience, resulting in a classic source- monitoring error.

Our findings apply more generally to how advertising affects consumers' cognitive processing after they have experienced a product. This is important because we know that consumers are likely to be exposed to advertising concerning products they have previously used. The majority of advertisements on the market today are for 'mature' products and serve to 'remind' consumers to purchase their product again. It is more likely, too, that an advertisement will be noticed after a consumer has had a direct experience (Lodish et al., 1995) because some consumers seem to justify their past purchase decisions by seeking out advertising (Cohen and Goldberg, 1970). The advertisement received post-experience may be used by consumers to rationalize, reconstruct or reinterpret past experiences. 
From the traditionally held consumer viewpoint, one's own experience drives decision making. Experience-based beliefs tend to be strong, confidently held and more highly correlated with intentions and buying behavior (Smith, 1993). Advertising, meanwhile, is disdained. Consumers know it is intended to manipulate, and they believe it has little effect on their decision making (Clark, 1985). However, this too could be an illusion, as Loftus and Pickrell (1995, p. 720) note, this type of situation may be ripe for memory distortion: 'New information invades us, like a Trojan horse, precisely because we don't detect its influence.'

\section{Acknowledgements}

The authors would like to thank all the reviewers as well as Dan Wright for their helpful comments on earlier drafts of this paper.

\section{References}

Armstrong, G. M., Gurol, M. N. and Russ, F. A. (1979). Detecting and correcting deceptive advertising. Journal of Consumer Research, 6, 237-246.

Barrett, P. M. (1995). Supreme Court says a distinctive color can be basis for a product trade mark. Wall Street Journal, 29 March, B10.

Belli, R. F. (1988). Color blend retrievals: Compromise memories or deliberate compromise responses? Memory \& Cognition, 16, 314-326.

Belli, R. F., Lindsay, D. S., Gales, M. S. and McCarthy, T. T. (1994). Memory impairment and source misattribution in postevent misinformation experiments with short retention intervals. Memory \& Cognition, 22, 40-54.

Burke, R. R., DeSarbo, W. W., Oliver, R. L. and Robertson, T. S. (1988). Deception by implication: An experimental investigation. Journal of Consumer Research, 14, 483-494.

Christiaansen, R. E. and Ochalek, K. (1983). Editing misleading information from memory: Evidence for the coexistence of original and postevent information. Memory \& Cognition, 11, 467-475.

Clark, E. (1985). The want makers: Inside the world of advertising. New York: Penguin Books.

Cohen, J. B. and Goldberg, M. E. (1970). The dissonance model in post-decision product evaluation. Journal of Marketing Research, 7, 315-321.

Deighton, J. (1984). The interaction of advertising and evidence. Journal of Consumer Research, 11, 763770. 
Donaldson, W. (1996). The role of decision processes in remembering and knowing. Memory \& Cognition, 24, 523-533.

Dwek, R. (1997). Compare and contrast. Management Today, March.

Greene, E., Flynn, M. S. and Loftus, E. F. (1982). Inducing resistance to misleading information. Journal of Verbal Learning and Verbal Behavior, 21, 207-219.

Harris, R. J. and Monaco, G. E. (1978). Psychology of pragmatic implication: Information processing between the lines. Journal of Experimental Psychology: General, 107, 122.

Jacoby, J. and Hoyer, W. D. (1982). Viewer miscomprehension of televised commercials: Selected findings. Journal of Marketing, 46, 12-26.

Johnson, M. K., Hashtroudi, S. and Lindsay, D. S. (1993). Source monitoring. Psychological Bulletin, 114, 3-28.

Lindsay, D. L. (1990). Misleading suggestion can impair eyewitnesses' ability to remember event details. Journal of Experimental Psychology: Learning, Memory \& Cognition, 16, 1077-1083.

Lockhart, R. S. (1979). Remembering events: Discussion of papers by Jacoby and Craik, Battig, and Nelson. In L. S. Cermak and F. I. M. Craik (Eds.), Levels of processing in human memory (pp. 7788). Hillsdale, NJ: Erlbaum.

Lodish, L. M., Abrahamson, M., Kalmenson, S., Livelsberger, J., Lubetkin, B., Richardson, B. and Stevens, M. E. (1995). How T.V. advertising works: A meta-analysis of 389 real world split cable T.V. advertising experiments. Journal of Marketing Research, 32, 124-139.

Loftus, E. F. (1977). Shifting human color memory. Memory \& Cognition, 5, 696-699.

Loftus, E. F. (1982). Memory and its distortions. From the G. Stanley Hall lecture series, Washington, DC: American Psychological Association.

Loftus, E. F. and Pickrell, J. E. (1995). The formulation of false memories. Psychiatric Annals, 25, 720-725. McCloskey, M. and Zaragoza, M. (1985). Misleading postevent information and memory for events: Arguments and evidence against memory impairment hypotheses. Journal of Experimental Psychology: General, 114, 1-16.

Pezdek, K. (1977). Cross-modality semantic integration of sentence and picture memory. Journal of Experimental Psychology: Human Learning and Memory, 3, 515-524.

Pezdek, K. and Greene, J. (1993). Testing eyewitness memory: Developing a measure that is more resistant to suggestibility. Law and Human Behavior, 27, 361-369.

Rajaram, S. (1993). Remembering and knowing: Two means of access to the personal past. Memory \& Cognition, 21, 89-102. 
Roediger, H. L. III and McDermott, K. B. (1995). Creating false memories: Remembering words not presented in lists. Journal of Experimental Psychology: Learning, Memory \& Cognition, 21, 803814.

Schacter, D. L. (1995). Memory distortion: History and current status. In D. L. Schacter, J. T. Coyle, G. D. Fischbach, M. M. Mesulam and L. E. Sullivan (Eds.), Memory distortion: How minds, brains and societies reconstruct the past. Cambridge, MA: Harvard University Press, $1 \pm 43$.

Sloan, A. E. (1996). The silent salesman. Food Technology, 50, 25.

Smith, R. E. (1993). Integrating information from advertising and trial: Processes and effects on consumer response to product information. Journal of Marketing Research, 30 (May), 204-219.

Tousignant, J. P., Hall, D. and Loftus, E. F. (1986). Discrepancy detection and vulnerability to misleading postevent information. Memory \& Cognition, 14, 329-338.

Tulving, E. (1985). Memory and consciousness. Canadian Psychologist, 26, 1-12.

Wilkie, W. L., McNeill, D. L. and Mazis, M. B. (1984). Marketing's `Scarlett Letter'. Journal of Marketing, 48, 11-31.

Wright, D. B., Varley, S. and Belton, A. (1996). Accurate second guesses in misinformation studies. Applied Cognitive Psychology, 10, 13-22.

Wright, D. B. (1993). Misinformation and warnings in eyewitness testimony: A new testing procedure to differentiate explanations. Memory, 1, 153-166.

Yamashita, M. (1996). A re-examination of the misinformation effect by means of visual and verbal recognition tests. Japanese Psychological Research, 38, 470-52. 